\title{
RENDIMENTO E ACÚMULO DE NUTRIENTES PELO ARROZ EM SOLUÇÃO NUTRITIVA COM E SEM A ADIÇÃO DE SILÍCIO(1)
}

\author{
L. S. SILVA(2) \& H. BOHNEN ${ }^{(3)}$
}

\begin{abstract}
RESUMO
O silício tem sido considerado benéfico para plantas acumuladoras do elemento, especialmente o arroz, justificando sua adição para o incremento da produção em solos deficientes. Entretanto, o efeito do Si, nestes casos, parece depender mais de determinadas condições ambientais e de efeitos indiretos, baseados nas características e reações no solo dos materiais normalmente utilizados como fontes de Si. Com o objetivo de avaliar o efeito do Si sobre o rendimento e sobre o acúmulo de macro e micronutrientes em arroz (Oryza sativa), cultivar BR-IRGA 410, foi realizado um estudo em casa de vegetação, com o cultivo de plantas em solução nutritiva completa, comparando os tratamentos com e sem a adição de $\mathrm{Si}$ na forma de $\mathrm{SiO}_{2}$ (em pó). Não houve diferença significativa entre as plantas com e sem adição de Si na produção de matéria seca da raiz, colmo + fol has e rendi mento de grãos, mas, sim, na produção de matéria seca de casca. As plantas que receberam $\mathrm{Si}$, em relação às plantas sem adição, apresentaram maior concentração de Si na raiz, colmo + folhas e casca, porém, foram similares no grão. A adição de Si na solução nutritiva diminuiu os teores de boro e fósforo nas raízes, e os teores de boro, cálcio, ferro e manganês, na casca. Não houve efeito significativo do Si nos teores de macro e micronutrientes no colmo + folhas e grãos.
\end{abstract}

Termos de indexação: raízes, colmo + folhas, cascas, grãos, casa de vegetação.

(1) Parte da Tese de Doutorado do primeiro autor, aprseentada ao Programa de Pós-Graduação em Ciência do Solo da U niversidade Federal do Rio Grande do Sul - UFRGS. Recebido para publicação em agosto de 2000 e aprovado em fevereiro de 2001.

(2) Engenheiro-Agrônomo, Dr. Pesquisador do IRGA/Convênio CAAL. Estação Experimental do Arroz. Setor de Solos e Águas. Av. Bonifácio C. Bernardes, 1494, CEP 94930-030 Cachoeirinha (RS). E-mail: Ieandro_irga@redemeta.com.br

(3) Engenheiro-Agrônomo, Colaborador convidado do Departamento de Solos, Faculdade de Agronomia, Universidade Federal do Rio Grande do Sul - UFRGS. Consultor do IRGA. E-mail: bohnen@conex.com.br 


\title{
SUMMARY: YIELD AND NUTRIENT ABSORPTION BY RICE GROWN IN NUTRIENT SOLUTION WITH AND WITHOUT SILICON
}

\begin{abstract}
Silicon has been consi dered a beneficial element for plants that accumulateit, specially rice, thus it is quitecommon its additi on to deficient soi ls. However, its effects seem to depend moreon environmental and indirect factors related to soil and silicon sources characteristics. With the objective to study the effect of silicon as the single growth factor of rice (Oryza sativa CV. BR-IRGA 410), an experiment was carried out using nutrient solution under greenhouse conditions, with and without the addition of silicon as $\mathrm{SiO}_{2}$ (powder). Results did not show yield increasein dry matter of roots, stem + leaves and grain. Only husk yield increased with silicon addition. With silicon addition to the nutrient solution, all parts of the plant showed higher concentration of silicon but not the grains. Silicon addition significantly reduced boron and phosphorus concentrations in theroots and boron, calcium, iron and manganeseconcentrations in thehusks. Silicon did not affect theconcentration of all other nutrients in the stem + leaves and grain.
\end{abstract}

Index terms: roots, stem + leaves, husks, grains, greenhouse

\section{NTRODUÇÃO}

O silício é um dos elementos mais abundantes da crosta terrestre, superado apenas pelo oxigênio (Heinen \& Oehler, 1979). Ele faz parte da composição da mai oria dos minerais, estando presente, em maior ou menor quantidade, em praticamente todos os tipos de solos. Na solução do solo, quando o pH do sistema situa-se abaixo de 9,0, encontra-se basicamente na forma de ácido monossilícico $\mathrm{Si}(\mathrm{OH})_{4}$, cuja concentração depende do produto de solubilidade dos diferentes pol imorfos de $\mathrm{SiO}_{2}$ que o sistema mineral pode apresentar, além do efeito de outros minerais presentes que contêm Si capazes de controlar sua solubilidade (Lewin \& Reimann, 1969; Drees et al., 1989). Segundo Elgawhary \& Lindsay (1972) e Drees et al. (1989), os valores da constante de equilíbrio para os óxidos de Si podem variar em torno de $10^{-4}$ para o quartzo até $10^{-2,74}$, para a sílica amorfa, de acordo com a reação de solubilidade $\mathrm{SiO}_{2}+2 \mathrm{H}_{2} \mathrm{O} \leftrightarrows \mathrm{Si}(\mathrm{OH})_{4}$.

$\mathrm{O} \mathrm{Si}(\mathrm{OH})_{4}$ é prontamenteabsorvido pelas plantas e, apesar de não ser considerado um el emento essencial, algumas espécies o absorvem em quantidades comparáveis ou bem superiores a macronutrientes essenciais (Marschner, 1995). Praticamente, todo o Si absorvido étranslocado das raízes para as folhas e, com a saída da água pela transpiração, pol imeriza na parte externa da parede celular (principalmente nas células da epiderme), transformando-se em um mineral amorfo de sílica denominado opala biogênica $\left(\mathrm{SiO}_{2} \cdot \mathrm{nH}_{2} \mathrm{O}\right)$ (Lanning et al., 1958). As plantas diferem quanto à absorção de $\mathrm{Si}$, sendo divididas em acumuladoras, intermediárias enão-acumuladoras, conforme ototal deSi absorvido ea quantidade do elemento fornecida por fluxo de massa (Marschner, 1995).
Apesar de ser a essencialidade às plantas superiores ainda questionada na literatura, o fornecimento de Si parece benéfico para o crescimento e produção de determinadas espécies, principalmente daquelas consideradas acumuladoras do elemento, tais como arroz e cana-deaçúcar (Lewin \& Reimann, 1969; Marschner, 1995).

Vários mecanismos apontados para explicar o efeito benéfico do Si encontram-se relacionados com ações indiretas provocadas pela deposição deSi nas fol has e com a conseqüente mai or rigi dez dos tecidos. As folhas tornam-se mais eretas, favorecendo a entrada de luz solar, maior absorção de $\mathrm{CO}_{2}$ e diminuição da transpiração excessiva, permitindo incremento da taxa fotossintética (Savant et al., 1997). A silificação das cél ulas epidérmicas também funcionaria como uma barreira física efetiva contra a penetração de hifas e contra o ataque de insetos, diminuindo os danos causados pela ocorrência de doenças e pragas (Lima Filho et al ., 1999a). Também há relatos de que o Si promove um efeito atenuante contra a toxidez deAl, Mn e Fe (Fischer et al., 1990; Ma et al., 1997; Savant et al., 1997).

Com base nestas considerações, diver sos autores têm estudado o efeito da adição de fontes de Si no cultivo de arroz com vistas em incrementar o rendimento da cultura (Yamauchi \& Winslow, 1989; Korndörfer et al., 1999). SegundoSavant et al. (1997), essa prática tem causado efeitos sobre a nutrição de N, P eK às plantas, não sendo possível distinguir o efeito isolado da disponibilidade de Si do efeito de outros fatores indiretos relacionados com a composi ção e reação no sol o dos materiais utilizados. Estes, muitas vezes, são subprodutos industriais que contêm entre 9,4 e $22 \%$ de Si com quantidades significativas de outros elementos, principalmente 
Ca e Mg, e queapresentam reação básica, alterando o pH do solo (Yoshida, 1981; Savant et al., 1997).

O objetivo deste trabalho foi avaliar o efeito isolado do Si sobre o rendimento e sobre o acúmulo de macro e micronutrientes em plantas de arroz cultivadas em solução nutritiva completa com esem a adição do elemento na forma de $\mathrm{SiO}_{2}$, como partículas extremamente pequenas, de modo a favorecer a cinética de dissolução da sílica e liberação de $\mathrm{Si}$, sem influir substancialmente em outras características da solução nutritiva.

\section{MATERIAL E MÉTODOS}

O experimento foi realizado em condições de casa de vegetação, no período de outubro de 1999 a fevereiro de 2000, no Departamento de Solos da Universidade Federal do Rio Grande do Sul, município de Porto Alegre (RS). Neste estudo, plântulas de arroz do cultivar BR-IRGA 410, com 13 dias de idade, foram transplantadas para vasos plásticos de $10 \mathrm{~L}$ (quatro plântulas por vaso suspensas em placa de isopor utilizada como vedação da parte superior dos vasos) que continham solução nutritiva completa (Quadro 1). Os tratamentos com e sem Si foram obtidos com a adição de $\mathrm{SiO}_{2}$ em pó (Silica fumed, 0,007 $\mu \mathrm{m}$, Sigma) na quantidade de $2 \mathrm{~g} \mathrm{vaso}^{-1}$.

A concentração de equilíbrio para oSi na sol ução nutritiva, determinada $24 \mathrm{~h}$ após a adição da sílica e sem a presença de plantas, indicou valores entre 8 e $12 \mathrm{mg} \mathrm{L}^{-1}$ de Si, enquanto a solução sem a adição de sílica apresentou teores menores que $0,1 \mathrm{mg} \mathrm{L}^{-1}$ de Si. Os tratamentos com e sem adição de Si, em delineamento completamente casualizado e com quatro repetições para cada tratamento, permaneceram expostos às variações detemperatura e umidade relativa do ambiente durante todo o período experimental, sendo o volume dos vasos completado com água destilada diariamente e a solução nutritiva renovada semanal mente.

Aos 47 dias do transplante, foram retiradas três plantas de cada vaso, mantendo-se a planta mais vigorosa. Durante cinco e sete dias (entre os 55 e 60 dias e entre os 88 e 95 dias após o transplante), foi avaliada a evapotranspiração por pesagem dos vasos e feito o cálculo do consumo de água em $24 \mathrm{~h}$. Aos 73 e 103 dias do transplante, foram col etadas quatro folhas, anteriores à fol ha bandeira, de cada planta de cada tratamento que, após colocadas em tubos dePVC (10 cm de altura e 2,5 cm de diâmetro) com papel filtro 0,2 $\mu \mathrm{m}$ versapor (Gelman Sciences Inc.) colado no fundo, foram congeladas a $-16^{\circ} \mathrm{C}$ por $24 \mathrm{~h}$ e, quando descongeladas, foram centrifugadas (30 min. a $5.000 \mathrm{rpm}$ ) para retirada do extrato celular eanálise imediata do teor deSi por absorção atômica.

Ao final de 120 dias, as plantas foram colhidas, secas em estufa a $60^{\circ} \mathrm{C}$, e os grãos trilhados manualmente e descascados mecanicamente. Nas plantas, foram avaliadas as seguintes características: número de panículas, produção de matéria seca das raízes, col mo +fol has e casca erendimento de grãos. Subamostras das raízes, colmo + fol has e casca foram moídas em moinho tipo "Willye" com peneira de malha de $1 \mathrm{~mm}$ de diâmetro, e dos grãos moídos em moinho defacas (Telator Knifetec 1095 Sample Mill) com velocidade do rotor de $20.000 \mathrm{rpm}$ em dois pulsos de $10 \mathrm{~s}$.

\section{Quadro 1. Concentração dos nutrientes e produtos utilizados no preparo da solução nutritiva}

\begin{tabular}{|c|c|c|}
\hline Elemento & Concentração & Produto (p.a.) \\
\hline \multicolumn{3}{|c|}{$\mathrm{mmol} \mathrm{L-1}$} \\
\hline $\mathrm{N}-\mathrm{NO}_{3}$ & 3,0 & $\mathrm{NH}_{4} \mathrm{NO}_{3}, \mathrm{Ca}\left(\mathrm{NO}_{3}\right) \cdot 4 \mathrm{H}_{2} \mathrm{O}$ \\
\hline $\mathrm{N}-\mathrm{NH}_{4}$ & 1,5 & $\mathrm{NH}_{4} \mathrm{H}_{2} \mathrm{PO}_{4}, \mathrm{NH}_{4} \mathrm{NO}_{3}$ \\
\hline $\mathrm{P}$ & 0,5 & $\mathrm{NH}_{4} \mathrm{H}_{2} \mathrm{PO}_{4}$ \\
\hline K & 2,0 & $\mathrm{KCl}$ \\
\hline $\mathrm{Ca}$ & 1,5 & $\mathrm{CaCl}_{2} .2 \mathrm{H}_{2} \mathrm{O}, \mathrm{Ca}\left(\mathrm{NO}_{3}\right)_{2} .4 \mathrm{H}_{2} \mathrm{O}$ \\
\hline $\mathrm{Mg}$ & 1,0 & $\mathrm{MgSO}_{4} .7 \mathrm{H}_{2} \mathrm{O}$ \\
\hline $\mathrm{S}$ & 1,0 & $\mathrm{MgSO}_{4} .7 \mathrm{H}_{2} \mathrm{O}$ \\
\hline B & 0,023 & $\mathrm{H}_{3} \mathrm{BO}_{3}$ \\
\hline $\mathrm{Cl}$ & 3,0 & $\mathrm{KCl}, \mathrm{CaCl}_{2} \cdot 2 \mathrm{H}_{2} \mathrm{O}$ \\
\hline $\mathrm{Cu}$ & 0,0047 & $\mathrm{CuSO}_{4} .5 \mathrm{H}_{2} \mathrm{O}$ \\
\hline $\mathrm{Fe}$ & 0,18 & Fe-EDTA $\left(\mathrm{FeSO}_{4} .7 \mathrm{H}_{2} \mathrm{O}+\mathrm{Na}_{2} \mathrm{EDTA}\right)$ \\
\hline $\mathrm{Mn}$ & 0,0047 & $\mathrm{MnSO}_{4} \cdot \mathrm{H}_{2} \mathrm{O}$ \\
\hline Mo & 0,00011 & $\mathrm{NaMoO}{ }_{4} \cdot 2 \mathrm{H}_{2} \mathrm{O}$ \\
\hline $\mathrm{Zn}$ & 0,0015 & $\mathrm{ZnSO}_{4} .7 \mathrm{H}_{2} \mathrm{O}$ \\
\hline
\end{tabular}

$\mathrm{O} \mathrm{pH}$ foi mantido entre 5,5 e 6,5 com $\mathrm{NaOH}$ 0,5 $\mathrm{mol} \mathrm{L}^{-1}$. 
Foi determinado o teor de Si por absorção atômica, após digestão do tecido com uma mistura de $\mathrm{HF}, \mathrm{HNO}_{3} \mathrm{eH}_{2} \mathrm{O}_{2}$ (250 mg de amostra, $0,5 \mathrm{~mL}$ de $\mathrm{H}_{2} \mathrm{O}, 5 \mathrm{~mL}$ de $\mathrm{HNO}_{3}, 0,5 \mathrm{~mL}$ de $_{2} \mathrm{O}_{2}$ e $2 \mathrm{~mL}$ de HF) em recipiente de teflon hermeticamente fechado e uso de microondas DET 100, com a seguinte programação de tempo (min): 5, 1, 4, 1, 3 nas respectivas potências (watts): 400, 790, 320, 790 e 000, conforme recomendação do fabricante (Provecto, s/d).

Também foram determinados, após digestão com $\mathrm{H}_{2} \mathrm{SO}_{4}, \mathrm{H}_{2} \mathrm{O}_{2}$ e mistura de digestão, os teores de $\mathrm{N}$ por destilação em semimicro Kjedhal, $\mathrm{P}$ por colorimetria, $\mathrm{K}$ por fotometria de chama e $\mathrm{Ca}$ e $\mathrm{Mg}$ por absorção atômica; após digestão com $\mathrm{HNO}_{3} \mathrm{e}$ $\mathrm{HClO}_{4}$, os teores de S por col orimetria, $\mathrm{Cu}, \mathrm{Fe}, \mathrm{Mn}$ e Zn por absorção atômica, e, após quei ma em mufla a $600^{\circ} \mathrm{C}$ por uma hora, o teor de B por colorimetria, conforme os métodos descritos em Tedesco et al. (1995). Todos os resultados para os parâmetros avaliados foram submetidos à análise da variância com a comparação das médias dos tratamentos com e sem adição de Si pelo F-teste $(P<0,05)$.

\section{RESULTADOS E DISCUSSÃO}

Não foram encontradas diferenças significativas entre a adição ou não de Si nas características avaliadas nas plantas, exceto no teor de Si dos extratos das fol has e na produção de matéria seca de casca (Quadro 2). Os teores de Si no extrato de fol has confirmaram a absorção pelas raízes e a transl ocação do el emento para a parte aérea ainda na forma solúvel para aquelas plantas cultivadas na solução nutritiva com a adição de Si. Essa diferença na concentração de Si também pôde ser detectada com as mãos, pela aspereza das fol has das plantas tratadas com adição de Si, durante todo o cido vegetativo. Segundo Rafi \& E pstein (1999), esse tipo de "teste do tato", embora subjetivo, permitiu diferenciar as plantas cultivadas em solução nutritiva com e sem a adição de Si e demonstrou a sua pol imerização na forma de uma estrutura rígi da nas folhas (opala biogênica). As maiores produções de matéria seca de casca obtidas para as plantas com a adição de Si mostraram-se relacionadas com o fato de ser este um compartimento da planta com maior deposição de Si (Savant et al., 1997).

Houve acúmulo significativo de Si nas raízes, colmo + fol has e casca com a adição deste elemento na solução nutritiva, o que não ocorreu com os grãos (Quadro 3). Entretanto, os valores obti dos na sol ução nutritiva ficaram abaixo daqueles normalmente encontrados em plantas cultivadas no solo (Savant et al., 1997), explicando em parte o fato de não ter sido encontrada diferença na evapotranspiração entreas plantas sem e com Si nos períodos avaliados (Quadro 2). Este comportamento na evapotranspiração, entretanto, foi um indicativo de que não houve influência no suprimento dos outros nutrientes por fluxo de massa entre os tratamentos com e sem Si. O maior teor de Si na casca também pôde ajudar a explicar a maior produção de matéria seca deste compartimento da planta (Quadro 2).

Quadro 2. Produção de matéria seca, número de panículas, rendimento de grãos, evapotranspiração e teor de Si no extrato de folhas de plantas de arroz cultivado em solução nutritiva com e sem a adição de silício

\begin{tabular}{|c|c|c|c|}
\hline Característica & Avaliação & Sem silício & Com silício \\
\hline \multirow[t]{3}{*}{ Produção de matéria seca (g planta-1) } & Raízes & $22,2 \mathrm{a}$ & 22,9 a \\
\hline & Colmo + folhas & 255,9 a & 258,5 a \\
\hline & Cascas & $44,4 \mathrm{~b}$ & 50,6 a \\
\hline N úmero de panículas (planta-1) & - & 59 a & $60 \mathrm{a}$ \\
\hline Rendimento de grãos (g planta-1) & - & 110,4 a & 118,5 a \\
\hline \multirow[t]{2}{*}{ Evapotranspiração (mL vaso-1 dia-1) } & 55 a 60 dias & $1.232 \mathrm{a}$ & $1.205 \mathrm{a}$ \\
\hline & 88 a 95 dias & $1.615 \mathrm{a}$ & $1.663 \mathrm{a}$ \\
\hline \multirow[t]{2}{*}{ Si extrato das folhas ( $\mathrm{mg} \mathrm{L}^{-1}$ ) } & 73 dias & $2,0 \mathrm{~b}$ & $34 \mathrm{a}$ \\
\hline & 103 dias & $0,8 \mathrm{~b}$ & $23 a$ \\
\hline
\end{tabular}

Média de quatro repetições, exceto Si extrato (duas repetições).

Médias seguidas de letras distintas, na mesma linha, diferem significativamente entre si pelo Teste-F $(P<0,05)$. 
Quadro 3. Teores de silício, macro e micronutrientes na raiz, colmo + folhas, casca e grãos de arroz cultivado em solução nutritiva com e sem a adição de silício

\begin{tabular}{|c|c|c|c|c|c|c|c|c|}
\hline \multirow{2}{*}{ Elemento } & \multicolumn{2}{|c|}{ Raiz } & \multicolumn{2}{|c|}{ Colmo + folha } & \multicolumn{2}{|c|}{ Casca } & \multicolumn{2}{|c|}{ Grão } \\
\hline & sem $\mathbf{S i}$ & $\operatorname{com} \mathbf{S i}$ & sem $\mathbf{S i}$ & $\operatorname{com} \mathbf{S i}$ & sem $\mathbf{S i}$ & $\operatorname{com} \mathrm{Si}$ & sem $\mathbf{S i}$ & com $\mathbf{S i}$ \\
\hline & & & & - $\mathrm{g}$ & & & & - \\
\hline $\mathrm{Si}$ & $0,8 \mathrm{~b}$ & $14,3 \mathrm{a}$ & $1,1 \mathrm{~b}$ & $15,0 \mathrm{a}$ & $0,8 \mathrm{~b}$ & $23,3 a$ & 3,3 & 3,5 \\
\hline $\mathrm{N}$ & 10,0 & 12,0 & 12,3 & 10,1 & 8,4 & 9,9 & 19,4 & 19,8 \\
\hline $\mathrm{P}$ & 3,4 a & $2,0 \mathrm{~b}$ & 2,4 & 1,8 & 2,0 & 2,1 & 4,5 & 4,2 \\
\hline K & 14,5 & 12,6 & 39,5 & 41,6 & 14,6 & 13,4 & 3,5 & 3,4 \\
\hline $\mathrm{Ca}$ & 1,5 & 1,5 & 4,4 & 3,6 & $1,5 \mathrm{a}$ & $1,0 \mathrm{~b}$ & 0,1 & 0,1 \\
\hline $\mathrm{Mg}$ & 1,0 & 1,0 & 3,7 & 2,7 & 1,5 & 1,4 & 1,7 & 1,6 \\
\hline s & 2,8 & 3,0 & 2,3 & 1,9 & 1,3 & 1,2 & 1,4 & 1,2 \\
\hline B & 20 & $110 \mathrm{~h}$ & 185 & ת & 1502 & $2 \mathrm{~h}$ & 13 & 11 \\
\hline $\mathrm{Cu}$ & 653 & 746 & 41,5 & 31,3 & 25,0 & 14,9 & $\begin{array}{r}1, \\
19,2\end{array}$ & $\begin{array}{r}1,4 \\
16,0\end{array}$ \\
\hline $\mathrm{Fe}$ & 5.648 & 2.840 & 550 & 424 & 98 a & $75 \mathrm{~b}$ & 27 & 24 \\
\hline $\mathrm{Mn}$ & 7,4 & 12,3 & 144 & 120 & $80 \mathrm{a}$ & $60 \mathrm{~b}$ & 15 & 14 \\
\hline $\mathrm{Zn}$ & 38 & 37 & 45 & 39 & 48 & 45 & 31 & 27 \\
\hline
\end{tabular}

Somente as médias com letras distintas, na mesma linha, e dentro de cada parte da planta avaliada, diferem significativamente entre si pelo Teste-F $(P<0,05)$.

O silício absorvido pelas plantas de arroz causou diminuição significativa dos teores de $\mathrm{P}$ e B nas raízes (Quadro 3). O desenvolvimento de aerênquima pelas plantas de arroz, como um mecanismo para tolerância à baixa tensão de oxigênio molecular em sol os alagados, permite a liberação de oxigênio dos tecidos arenquimáticos para o solo, que pode ser utilizado para oxidar Fe e Mn (Matta, 1999).

Segundo van der Vorm (1980), a maior preci pitação de Fe nas raízes de plantas de arroz foi resultado do maior suprimento de Si na solução nutritiva. Kluthcouski \& Nel son (1980) encontraram decréscimo da concentração de $M n$ nas fol has de soja com a adição de Si, mas as raízes não foram afetadas. Para o arroz, H origuchi (1988) demonstrou aumento da concentração de $\mathrm{Mn}$ nas raízes para o tratamento com adição de Si, atribuído à maior oxidação e deposição do el emento na superfície das raízes. O P, pela sua alta afinidade com Fe e Mn, também teria sua disponibilidade na solução e, ou, absorção pelas plantas afetadas pelas alterações provocadas pelo Si na disponibilidade destes elementos ( $\mathrm{Ma}$ \& Takahashi, 1990).

Embora as diferenças nos teores de Fe e $M n$ não tenham sidosignificativas, os resultados encontrados para oP, juntamente com os da literatura, indicaram um efeito do $\mathrm{Si}$, mas não esclareceram qual o mecanismo afetado. Também tem sido sugerido que o Si promoveria maior "poder oxidante" às raízes, em decorrência do maior volume e rigidez dos aerênquimas e, ou, maior atividade de enzimas peroxidases (Horiguchi, 1988; Marschner, 1995).

Alterações no teor de B em função da absorção de Si não foram encontradas na literatura, mas Marschner (1995) ressaltou as similaridades quími cas doáci do silícico e do ácido bórico em solução e o fato de que o B, juntamente com o Ca, participa da síntese e estruturação da parede celular das células e que ambos apresentam demanda inversamente proporcional à capacidade de absorver $\mathrm{Si}$, quando comparadas espécies de monocotil ledôneas com dicotiledôneas.

A absorção de Si pelas plantas não alterou significativamente os teores dos macro e micronutrientes no colmo + fol has em comparação com as plantas sem adição do el emento (Quadro 3), apesar de terem sido os val ores absolutos dos teores quase sempre menores para o tratamento com Si. Comportamento diferente foi encontrado na casca, compartimento da planta que apresentou a maior concentração de Si com a sua adição na solução nutritiva, com um correspondente decréscimo significativo nos teores de Ca, B, Fee Mn (Quadro 3).

$O$ efeito do Si sobre o teor de $B$ foi observado nas raízes, o que deveter refletido na definição dos teores deste compartimento. Embora, neste trabalho, as diferenças não tenham sido significativas para Fee Mn nas raízes, Ma \& Takahashi (1990) demonstraram o efeito do Si sobre a absorção deFe e Mn, alterando sua concentração nos órgãos aéreos da planta. 
Embora o col mo + fol has apresentassem menor val or para o teor de Ca nas plantas com $\mathrm{Si}$, em relação àquelas sem $\mathrm{Si}$, as diferenças somente foram significativas na casca, justamente onde foram obtidos os mai ores teores deSi. I nanaga et al . (1995) encontraram decréscimo nos teores de Ca no tecido, atribuído a efeitos da adição de Si na absorção deste elemento pelas plantas de arroz.

Conforme abordado anteriormente, existe uma relação inversa entre a necessidade de B e Ca na formação da estrutura da parede celular e a capacidade de absorver Si pelas monocotiledôneas em relação a dicotiledôneas (Marschner, 1995).

O grão teve um comportamento bastante distinto dos outros compartimentos, pois não houve efeito significativo da adição de Si no acúmulo de macro e micronutrientes em plantas de arroz cultivadas em solução nutritiva com e sem a adição do el emento, inclusive para o próprio Si (Quadro 3). Tal comportamento pode significar uma espécie de sel eção por parte da planta de todos os nutrientes a serem depositados neste compartimento, independentemente da quantidade total absorvida. Deve-se consi derar também quea distribuição do Si nos órgãos aéreos da planta está diretamente relacionada com a taxa de transpiração específica de cada órgão, responsável pela polimerização do silício na forma de sílica (J ones \& Handreck, 1967; Marschner, 1995).

Apesar de absorvido pelas plantas em grande quantidade, o Si não apresentou efeito esperado e relatado na literatura sobre os componentes de produção de matéria seca da parte aérea e de rendimento de grãos do arroz. Tal comportamento poderia estar relacionado com as condições em que o experimento se desenvolveu, ou seja, sem a ocorrência de algum estresse de ordem nutricional ou ambiental significativo, os quais ocorrem normalmente com o cultivo no campo.

O principal argumento para as plantas não apresentarem uma resposta positiva à adição de $\mathrm{Si}$ nestas condições, embora o tenham absorvido em quantidades relativamente grandes para a dose de Si utilizada, talvez resida no fato que ainda não foi comprovado que oSi apresenta uma função biológica específica nas plantas. Quimicamente, o Si é muito parecido com o carbono, mas dificilmente participaria de al gum sistema metaból ico, pois este el emento tendea formar ligações mais estáveis com o oxigênio do que com hidrogênio em comparação com o carbono (Allinger et al., 1976).

Dessa forma, os compostos deSi cuja unidade éa $\mathrm{SiO}_{2}$ normalmente são muito pouco solúveis, reduzindo sua dinâmica na natureza. Isto permite sugerir que os efeitos de fontes de Si encontrados na literatura sobre o rendimento de arroz cultivado no campoe atribuídos ao aumento da disponi bi lidade de Si no solo parecem estar mais relacionados com fatores de natureza indireta, principalmente envolvendo desequilíbrios de ordem nutricional ou ambiental.

Embora diferenças no acúmulo de alguns nutrientes tenham sido detectadas pela presença do Si (Quadro 3), não foi possível distinguir, neste trabalho, se o efeito do Si ocorreu sobre a disponibilidade ou absor ção destes nutrientes ou em al guma utilização diferenciada no metabolismo da planta. Uma constatação importante é que essas diferenças provocadas pela adição do Si não se refletiram nos componentes de produção de matéria seca e de rendimento de grãos (Quadro 2). Outros estudos mais específicos, principalmente com relação à influência do Si sobre a dinâmica de absorção do $\mathrm{Fe}, \mathrm{Mn}, \mathrm{B}, \mathrm{P}$ e Ca, são necessários para esclarecer esses questionamentos.

Os resultados revelaram ausência de efeito do Si norendimento dearroz, parecendo contraditórios em relação aos encontrados na literatura que sugerem a adição de fontes de Si para a cultura. Entretanto, estes trabalhos buscam resposta para a adição de Si em solos que foram submetidos à elevada intemperização e percolação de nutrientes, que, normalmente, tendem a apresentar baixos níveis do elemento e de outros nutrientes essenciais e, portanto, de baixa fertilidade natural. Além disso, como foi abordado anteriormente, em condições de campo, as plantas estão mais sujeitas ao ataque de insetos e patógenos onde o efeito benéfico do Si é mais evidente.

Outro fator a ser considerado nestes estudos é que foram utilizados como principais fontes deSi os metassilicatos de $\mathrm{Na}$ e $\mathrm{K}$ e as escórias básicas de siderurgia (silicatos de Ca e Mg) (Lima Filho, 1999b), que podem conter como "impurezas" outros elementos essenciais às plantas e que podem encontrar-se em deficiência nos solos utilizados, especialmente Ca, Mg e Mn (Yoshida, 1981). Esses materiais ainda funcionam como corretivos dos efeitos da acidez em sua reação básica no solo (Yoshida, 1981), podendo a alteração do $\mathrm{pH}$ influenciar a disponibilidade dos nutrientes. Além disso, o silício liberado também poderia competir pel os síti os de adsorção específica no solo, deslocando o P retido na superfície de óxidos para a solução do sol o (Savant et al., 1997; Carval ho et al., 2000).

Mesmo naqueles trabalhos em que se utilizou solução nutritiva, para J ones \& Handreck (1967), os tratamentos com adição deSi somenteapresentariam resposta por eventuais desbalanços nutricionais na solução empregada, especialmente com relação à ocorrência de toxidez de Mn. N estes casos, também deve ser considerada a necessi dade de compensação dos teores de outros el ementos ou de corr eção do pH diferenciadas entre os tratamentos com esem Si pelo uso defontes deSi queapresentamíons acompanhantes ou de reação básica na solução nutritiva, o que nãofoi ocaso do material utilizado nesteexperimento. 


\section{CONCLUSÃO}

A adição de silício para plantas de arroz cultivadas em solução nutritiva diminuiu os teores de boro e fósforo nas raízes, de boro, cálcio, ferro e manganês na casca e aumentou a produção de matéria seca de casca, mas não alterou a produção de matéria seca das raízes, colmo + folhas e o rendimento de grãos.

\section{AGRADECIMENTO}

Os autores agradecem ao Laboratório deAnálises de Solos e Plantas da Faculdade de Agronomia da UFRGS, pela realização das análises de tecido neste trabalho.

\section{LITERATURA CITADA}

ALLINGER, N.L.; CAVA, M.P.; J ONGH, D.C.; J OHNSON, C.R.; LEBEL, N.A. \& STEVENS, C.L. Química orgânica. 2.ed. New York, Worth Publishers, 1976. 961p.

CARVALHO, R.; FURTINI NETO,A.E.; CURI, N.;FERNANDES, L.A. \& OLIVEIRA J r., A.C. Dessorção de fósforo por silício em sol os cultivados com eucalipto. R. Bras. Ci. Solo, 24:6974, 2000.

DREES, L.R.; WILDING, L.P.; SMECK, N.E. \& SENKAYI, A.L. Silica in soils: quartz and disordered silica polymorphs. In: DIXON, J.B. \& WEED, S.B., eds. Mineral in soil environments. 2.ed. Wisconsin, Soil Science Society of American, 1989. p.913-974.

ELGAWHARY, S.M. \& LINDSAY, W.L. Solubility of silica in soils. Soil Sci. Soc. Am. Proc., 36:439-442, 1972.

FISCHER, R.G.; EIMORE, I.E.; MILAN, P.A. \& BISSANI, C.A. Efeito do cal cário e fontes de silício sobre a toxidez de ferro em arroz irrigado. Lav. Arrozeira, 43:6-10, 1990.

HEINEN,W. \& OEHLER, J.H. Evolutionary aspects of biological involvement in the cycling of silica. In: TRUDINGER, P.A. $\&$ SWAINE, D.J ., eds. Biogeochemical cyding of mineralfarming elements. Amsterdan, Elsevier, 1979. p.431-441.

HORIGUCHI, T. Mechanism of manganese toxicity and tolerance of plants IV. Effects of silicon on alleviation of manganese toxicity of rice plants. Soil Sci. Plant N utr., 34:65-73, 1988.

INANAGA, S.; OKASAKA, A. \& TANAKA, S. Does silicon exist in association with organic compounds in rice plant? Soil Sci. Plant Nutr., 41:111-117, 1995.

J ONES, L.H.P. \& HANDRECK, K.A. Silica in soils, plants, and animals. Adv. Agron., 19:107-149, 1967.
KLUTHCOUSKI, J . \& NELSON, L.E. The effect of silicon on the manganesenutrition of soybeans (Glycinemax (L.) Merril). Plant Soil, 56:157-160, 1980.

KORNDÖRFER, G.H.; ARANTES, V.A.; CORREA, G.F. \& SNYDER, G.H. Efeito do silicato de cálcio no teor de silício no solo e na produção de grãos de arroz de sequeiro. R. Bras. Ci. Solo, 23:635-641, 1999.

LANNING, F.C.; PONNAIYA, B.W.X. \& CRUMPTON, C.F. The chemical nature of silica in plants. Plant Physiol., 33:339343, 1958.

LEWIN, J. \& REIMANN, B.E.F. Silicon and plant growth. Ann. Rev. Plant Physiol., 20:289-304, 1969.

LIMA FILHO, O.F.; LIMA, M.T.G. \& TSAI, S.M. O silício na agricultura. Inf. Agron., 87:1-7,1999b. (encarte técnico)

LIMA FILHO, O.F.; LIMA, M.T.G. \& TSAI, S.M. Supressão de patógenos em solos por agentes abióticos: o caso do silício. Inf. Agron., 87:8-12, 1999a. (encarte técnico)

MA, J.F. \& TAKAHASHI, E. Effect of silicon on the growth and phosphorus uptake of rice. Plant Soil, 126:115-119, 1990.

MA,J .F.; SASAKI, M. \& MATSUMOTO, H. Al-induced inhibition of root elengation in corn, Zea mays $\mathrm{L}$. is overcome by $\mathrm{Si}$ addition. Plant Soil, 188:171-176, 1997.

MARSCHNER, H. Mineral nutrition of higher plants. 2.ed. San Diego, Academic Press, 1995. 889p.

MATTA, F.M. Mecanismos fisiológicos associados ao desenvolvimento do sistema radicular das plantas. In: FERNANDES, M.F.;TAVARES, E.D. \& LEAL, M.L.S., eds. Workshop sobre Sistema Radicular: Metodologias e Estudo deCasos, Aracajú, 1999. Anais. Aracaju, Empresa Brasileira de Pesquisa Agropecuária. 1999. p.19-45.

PROVECTO. Métodos para digestão de amostras. Provecto Sistemas Analíticos, s.d. 220p.

RAFI, M.M. \& EPSTEIN, E. Silicon absortion by wheat (Triticum aestivum L.). Plant Soil, 211:223-230, 1999.

SAVANT, N.K.; SNYDER, G.H. \& DATNOFF, L.E. Silicon management and sustainable rice production. Adv. Agron., 58:151-199, 1997.

TEDESCO, M.J .; GIANELLO, C.; BISSANI, C.A.; BOHNEN, H. \& VOLKWEISS, S.J. Análises de solo, planta e outros materiais. 2.ed. Porto Alegre, Universidade Federal do Rio Grande do Sul, 1995. 174p. (Bol etim técnico, 5)

van der VORM, P.D.J. Uptake of Si by five plant species, as influenced by variations in Si-supply. Plant Soil, 56:153156,1980

YAMAUCHI, M. \& WINSLOW, M.D. Effect of silica and magnesium on yield of upland rice in the humid tropics. Plant Soil, 113:265-269, 1989.

YOSHIDA, S. Fundamentals of rice crop science. Manila, The International Rice Research Institute, 1981. 269p. 
